\title{
Tumor odontogênico queratocístico com transformação ameloblástica: relato de caso
}

\author{
Ameloblastic transformation of a keratocystic odontogenic tumor: case report
}

\section{Tumor odontogénico queratoquístico de transformación ameloblástica: reporte de um caso}

\author{
Danilo Chizzolini MASOCATTO ${ }^{1}$ \\ Murilo Moura OLIVEIRA ${ }^{1}$ \\ Gustavo Silva PELISSARO² \\ Janayna Gomes Paiva OLIVEIRA ${ }^{3}$ \\ Cauê Monteiro dos SANTOS ${ }^{4}$ \\ Juliana Andrade MACENA ${ }^{4}$ \\ Fernando Ribeiro TEIXEIRA ${ }^{4}$ \\ José Carlos Garcia de MENDONÇA ${ }^{5}$ \\ Ellen Cristina GAETTI-JARDIM ${ }^{6}$
}

\author{
Especialista em Cirurgia e Traumatologia Bucomaxilofacial (CTBMF): Implantodontista· Mestrando em Odontologia, \\ Faculdade de Odontologia do Mato Grosso do Sul (FAODO)/UFMS, Campo Grande - MS, Brasil
${ }^{2}$ Cirurgião Bucomaxilofacial (Hospital Municipal do Tatuapé/SP); Mestre em Clínica Odontológica; \\ Preceptor da Residência em CTBMF do Hospital Universitário "Maria Aparecida Pedrossian" / UFMS, Campo Grande - MS, Brasil \\ ${ }^{3}$ Especialista em Cirurgia e Traumatologia Bucomaxilofacial (CTBMF); Mestre em Ciências da Saúde; Doutoranda em Ciências da Saúde; \\ Preceptora da Residencia em CTBMF do Hospital Universitário "/ UFMS, Campo Grande - MS, Brasil \\ ${ }^{4}$ Residente em Cirurgia e Traumatologia Bucomaxilofacial (CTBMF), Núcleo de Hospital Universitário "Maria Aparecida Pedrossian”, UFMS, Campo Grande - MS, Brasil \\ ${ }^{5}$ Especialista em Cirurgia e Traumatologia Bucomaxilofacial (CTBMF); Mestre e Doutor em Odontologia; \\ Docente de Cirurgia da Faculdade de Odontologia do Mato Grosso do Sul (FAODO)/UFMS, Campo Grande - MS, Brasil (FAODO)/UFMS, Campo Grande - MS, Brasil \\ ${ }^{6}$ Especialista, Mestre e Doutora em Cirurgia e Traumatologia Bucomaxilofacial, Faculdade de Odontologia de Araçatuba, UNESP, \\ Mestre e Especialista em Estomatologia; Docente de Cirurgia da Faculdade de Odontologia do Mato Grosso do Sul (FAODO)/UFMS, Campo Grande - MS, Brasil
}

\begin{abstract}
Resumo
O tumor odontogênico queratocístico (TOQ) é uma patologia benigna, que apresenta controvérsias em seu diagnóstico e tratamento. Ocorre principalmente em região de ângulo mandibular, podendo estar ou não relacionada a um elemento dentário, comportamento agressivo e alta taxa de recorrência. A descoberta destas lesões muitas vezes é dada em exames de rotina, já que a maioria das lesões é assintomática e o diagnóstico definitivo é dado somente através do anatomohistopatológico. O objetivo deste trabalho é direcionar a melhor conduta ao cirurgião diante do tumor odontogênico de maior relevância clínica na odontologia através de diferentes formas de tratamento. Paciente do sexo feminino, 16 anos, feoderma, atendida no Hospital Universitário Maria Aparecida Pedrossian - UFMS, pela equipe de Cirurgia e Traumatologia Buco Maxilo-Facial acometida por um tumor odontogênico queratocístico com transformação ameloblástica em região posterior de mandíbula tratado com ressecção segmentar. A reconstrução no mesmo tempo operatório utilizou enxerto ósseo autógeno de crista ilíaca. Após quatro meses de acompanhamento observou-se oclusão satisfatória, sem queixas álgicas, sem mobilidade da placa de reconstrução, taxa de reabsorção do enxerto com valores normais. $\mathrm{O}$ tratamento dos ameloblastomas deve ser individualizado de acordo com tamanho da lesão, localização anatômica, idade do paciente, agressividade da mesma e imagem radiológica, sendo que as lesões multicísticas devem ser submetidas a ressecções mais amplas, com tecido sadio como margem de segurança para evitar recidivas. Portanto, não se deve advogar um único tipo de tratamento para pacientes com ameloblastoma. Descritores: Ameloblastoma; Diagnóstico; Etiologia; Terapêutica.
\end{abstract}

\section{Abstract}

The keratocystic odontogenic tumor (OKT) is a benign pathology, which is controversial in its diagnosis and treatment. It occurs mainly in mandibular angle region, which may or may not be related to a tooth, aggressive behavior and high recurrence rate. The discovery of such injuries is often given in routine examinations, since most of the lesions are asymptomatic and a definitive diagnosis is only given by anatomopathological and histological observations. The objective of this work is to direct the best approach to the surgeon at treatment of the most clinically relevant dental tumors in dentistry, with evidence about the forms of treatment and pointing the different types of autogenous bone graft. A female darkskinned patient, 16 year old, was attended at the University Hospital Maria Aparecida Pedrossian - UFMS by the team of Surgery and Traumatology Buco Maxillofacial affected by a keratocystic odontogenic tumor with ameloblastic transformation in the posterior mandible. The lesion was treated with segmental resection and reconstruction using autologous bone graft from the iliac crest. After four months of monitoring it was observed satisfactory occlusion, without pain complaints mobility or reconstruction plate mobility and the graft resorption rate within the normal range. Treatment of ameloblastoma should be individualized according to lesion size, anatomical location, patient age, aggressiveness and radiological imaging. The multicystic lesions should be subjected to more extensive resections with safety margin to prevent relapse. Therefore, one should not advocate a single type of treatment for patients with ameloblastoma.

Descriptors: Ameloblastoma; Diagnosis; Etiology; Therapeutics.

\section{Resumen}

El tumor odontogénico queratoquístico (TOQ) es una patología benigna, que es objeto de controversia en su diagnóstico y tratamiento. Se produce principalmente en la región de ángulo mandibular, que puede o no puede estar relacionado con un diente, el comportamiento agresivo y alta tasa de recurrencia. El descubrimiento de este tipo de lesiones se da a menudo en los exámenes de rutina, ya que la mayoría de las lesiones son asintomáticas y el diagnóstico definitivo sólo se da por anatomopatológico e histológico. El objetivo de este trabajo es dirigir el mejor enfoque para el cirujano antes de que los tumores dentales más clínicamente relevantes en odontología, discutir sobre las formas de tratamiento y exponer diferentes tipos de injerto óseo autógeno. Un paciente de sexo femenino, 16, feoderma, atendidos en el Hospital Universitario de Maria Aparecida Pedrossian - UFMS, el equipo de Cirugía y Traumatología Buco Maxilofacial afectado por un tumor odontogénico keratocystic con ameloblástica transformación en la mandíbula posterior tratado con resección segmentaria. La reconstrucción durante el mismo procedimiento que utilizó injerto óseo autógeno de la cresta ilíaca. Después de cuatro meses de seguimiento de la misma es con una oclusión satisfactoria y sin quejas de dolor y sin placa de reconstrucción movilidad, la tasa de reabsorción del injerto dentro del margen que se considera normal. El tratamiento del ameloblastoma debe ser individualizada según el tamaño de la lesión, localización anatómica, la edad del paciente, la agresividad de la misma y radiológica de imágenes, y las lesiones multiquísticos debe ser sometido a resecciones más extensas con el tejido sano como margen de seguridad contra recaídas. Por lo tanto, no hay que abogar por un solo tipo de tratamiento para pacientes con ameloblastoma

Descriptores: Ameloblastoma; Diagnóstico; Etiología; Terapéutica.

\section{INTRODUÇÃO}

O ceratocisto odontogênico foi, durante muito tempo considerado um cisto odontogênico de desenvolvimento com características especiais, já que apresenta comportamento clínico e histopatológico específicos. Em 2005 a OMS considera a referida patologia um tumor odontogênico e não, simplesmente uma lesão cística, sendo desde então designado como Tumor Odontogênico Queratocístico (TOQ). Acreditase que o TOQ se origine de remanescentes da lâmina dentária, embora outros autores apontem como origem as células da camada basal do epitélio oral adjacente à lesão ou ainda a partir da proliferação de pequenos hamartomas epiteliais do epitélio gengival. O TOQ apresenta um 
mecanismo de crescimento diferenciado, o qual se relaciona a fatores inerentes ao próprio epitélio, ainda não totalmente elucidados, ou à atividade enzimática na cápsula fibrosa ${ }^{1}$.

O ameloblastoma é o tumor odontogênico de maior significado clínico, sua frequência se iguala à de todos os outros tumores odontogênicos, excluindo os odontomas. Suas características histológicas quase sempre são benignas, com predominância nos ossos da mandíbula. No entanto, ele tem uma forte tendência à recorrência após a remoção cirúrgica conservadora. A ocorrência extra-óssea é incomum. São neoplasias do epitélio odontogênico e podem se originar dos ramanescentes da lâmina dentária, do órgão do esmalte em desenvolvimento, do revestimento epitelial de um cisto odontogênico ou das células da camada basal da mucosa bucal $^{2}$. Sua incidência é estimada em 0,5 por milhão de habitantes ao ano, embora em algumas partes do mundo, por exemplo, África do Sul, uma maior incidência foi relatada ${ }^{3}$.

Clinicamente, o tumor apresenta-se muitas vezes como um edema de forma assintomática, crescimento lento, em região posterior da mandíbula, sendo frequentemente associada a um dente incluso. A maioria dos pacientes tem entre 30 e 60 anos quando a lesão é diagnosticada. Não há predileção por sexo. Ocorrência múltipla é extremamente rara. Ao exame radiográfico pode se apresentar como uma lesão radiolúcida unilobular ou multilobular. Septos ósseos podem resultar em uma aparência de "favo de mel" ou "bolhas de sabão". Reabsorção de raízes pode ou não estar presente. O diagnóstico diferencial radiológico inclui uma variedade de cistos e tumores odontogênicos, particularmente o tumor odontogênico queratocístico, bem como cistos nãoodontogênicos e tumores, como uma lesão central de células gigantes, lesões fibro-ósseas e cisto ósseo simples. Foi mencionado que o ameloblastoma desmoplásico é muitas vezes caracterizado por uma radiografia com padrões de radiopacidade/radiolucência mista, com margens difusas, sugerindo uma lesão fibro-óssea ${ }^{4}$.

A melhor forma de tratamento é controversa. Esta neoplasia tende a se infiltrar entre as trabéculas ósseas do osso esponjoso na periferia da lesão, antes de uma reabsorção óssea tornar-se evidente radiograficamente. Portanto, a verdadeira margem do tumor, muitas vezes, estende-se além da aparente margem clínica ou radiográfica. A tentativa de remover o tumor por curetagem pode deixar pequenas ilhas do tumor no osso, que mais tarde se manifestam como recorrências ${ }^{5,6}$.

A ressecção marginal é a forma mais comum de tratamento, porém recorrências de $15 \%$ têm sido relatadas. Esta técnica minimiza o defeito da mandíbula, entretanto só pode ser aplicada em casos selecionados ${ }^{7}$. Muitos preconizam que a margem de segurança deve ser de pelo menos $1 \mathrm{~cm}$ além dos limites radiográficos do tumor ${ }^{8,9}$. Outros preconizam a ressecção segmentar ou em bloco, que permite remoção total da lesão e taxa de recorrência mais baixa.

\section{REVISÃO DA LITERATURA}

Sob o ponto de vista do diagnóstico diferencial, cistos e tumores odontogênicos com proliferação de epitélio odontogênico, podem apresentar aspectos semelhantes. Os tumores odontogênicos queratocísticos e os ameloblastomas são mais agressivos e podem recidivar quando submetidos a tratamento conservador. Os cistos dentígeros são menos agressivos que os tumores odontogênicos queratocísticos e os ameloblastomas, porém tem grande importância por serem mais comuns dos cistos odontogênicos além de que existe a hipótese de esse cisto transformar-se em ameloblastoma ${ }^{10,11}$ devido a possibilidade do gene p53, que está situado no braço curto do cromossomo 17, ser um regulador negativo do ciclo celular durante a oncogênese ${ }^{12}$.

Segundo Mendonça et al. ${ }^{13}$ os enxertos ósseos podem ser obtidos tanto de áreas intra-orais (mento, ramo, processo coronóide, borda inferior da mandíbula, zigoma e corpo mandibular) como de áreas extra-orais (crânio, tíbia, fíbula, costela, crista ilíaca). Os primeiros são facilmente obtidos, pertencem muitas vezes ao mesmo campo cirúrgico, mas para as grandes reconstruções dos maxilares são limitadas pelo pouco volume ósseo conseguido. Já os enxertos extra-orais vem ganhando cada vez mais importância e aplicabilidade nas grandes reconstruções maxilofaciais ${ }^{14}$.

Segundo Ahlmann et al. ${ }^{15}$ o ílio é o sítio doador preferido para os enxertos ósseos na reconstrução da região facial. Eles possuem a mesma origem embrionária que a mandíbula e podem ser obtidos tanto das porções anterior e posterior da crista ilíaca. Contém o maior volume ósseo medular e a proporção mais alta de osso medular/cortical. As complicações relatadas do sítio doador incluem distúrbios na marcha, hematoma, injúrias nervosas e arteriais, fraturas da asa do ilíaco, perfuração peritoneal, infecção, dor e um a taxa de reabsorção de $25 \%$ a $35 \%$ no primeiro ano.

Ao comparar enxertos vascularizados e não vascularizados, com relação ao sucesso do enxerto e à osseointegração de implantes colocados nos mesmos, os resultados são semelhantes até $6 \mathrm{~cm}$, o padrão de insucesso de enxertos não vascularizados aumenta naqueles maiores de $6 \mathrm{~cm}$ e o uso de enxerto não vascularizado deve ser usado com extrema precaução em defeitos que excedem o comprimento de $9 \mathrm{~cm}^{16,17}$.

\section{CASO CÍNICO}

Paciente feoderma, 16 anos, gênero feminino compareceu a clínica de cirurgia da faculdade de odontologia da UFMS para avaliação de $3^{\text {os }}$ molares. Ao exame físico extrabucal, não observou-se nenhuma assimetria em face. No exame intrabucal constatou-se ausência dos elementos 18, 28 , 38, 47 e 48, mucosas íntegras, sem presença de inflamação, exudato nem tampouco tumefação. A radiografia panorâmica revelou uma lesão radiolúcida multilocular, de limites bem definidos e halo esclerótico na região posterior direita de mandíbula, se estendendo de $1^{\circ}$ pré-molar a ramo ascendente com ausência de sintomatologia dolorosa (Figura 1).

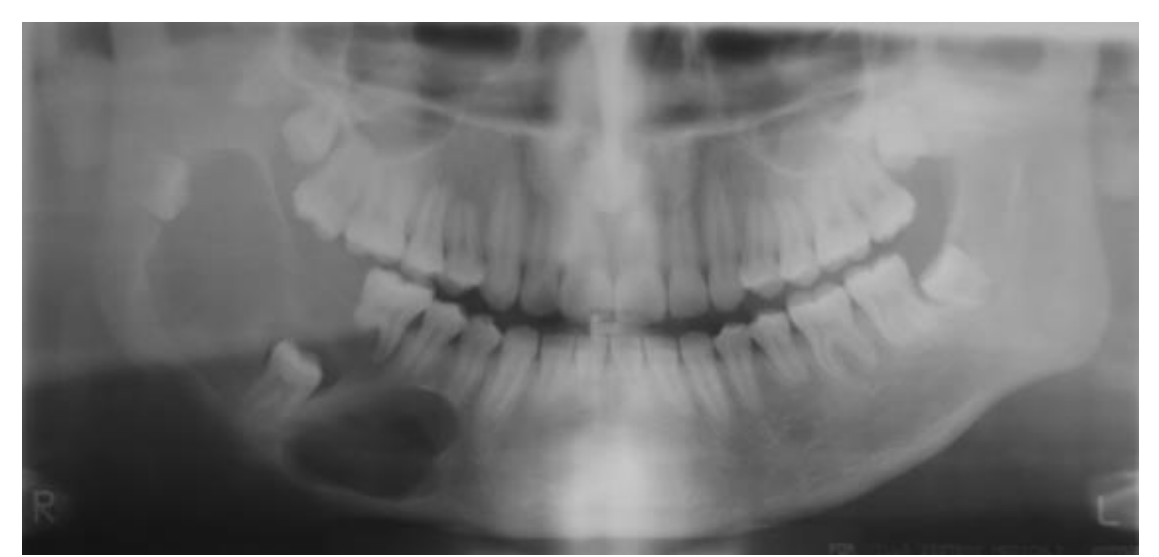

Figura 1: Radiografia panorâmica. Notar extensa lesão radiolúcida multilocular de limites bem definidos e halo esclerótico, na região posterior direita de mandíbula.

Em virtude das características mencionadas optou-se pelo planejamento cirúrgico de descompressão e biópsia incisional da lesão em questão, sob anestesia local. Durante o procedimento a punção revelou líquido amarelado com possíveis cristais de ceratina. Realizou-se uma incisão circular em fibromucosa da região do dente 48 , seguido de ostectomia e remoção de parte superior da cápsula da lesão, 
que foi enviada para o exame anatomohistopatológico.

A descompressão foi realizada colocando-se um dispositivo (segmento de sonda Folley) que foi posicionado no interior da cavidade com objetivo de manter comunicado o interior da lesão com a cavidade oral, e suturado nas margens da lesão com nylon 5-0. O exame anatomohistopatológico revelou se tratar de um tumor odontogênico queratocístico. No acompanhamento radiográfico de 6 meses notou-se a migração dos elementos dentários envolvidos na lesão em direção ao processo alveolar. Optou-se pelas exodontias dos elementos 47 e 48 .

Aos 8 meses de pós-operatório observou-se aumento volumétrico da região de corpo e ângulo mandibular direito, com expansão das corticais ósseas, reabsorção da raiz distal do elemento 46 e mobilidade dos elementos dentários 44, 45 e 46. Realizou-se nova biópsia incisional do local que revelou no exame anatohistopatológico de cisto dentígero.

Optou-se por nova descompressão mantendo-se o dispositivo por 15 dias pós-operatórios. Aos 3 meses pósoperatório, a paciente retornou com queixa de dor e com aumento de volume da região, gerando assimetria facial direita (Figura 2). Optou-se por nova biópsia com coleta de material dos planos mais profundos e marsupialização da referida lesão com envio da peça para nova análise anatomohistopatológica que revelou se tratar de ameloblastoma multicístico (Figura 3).

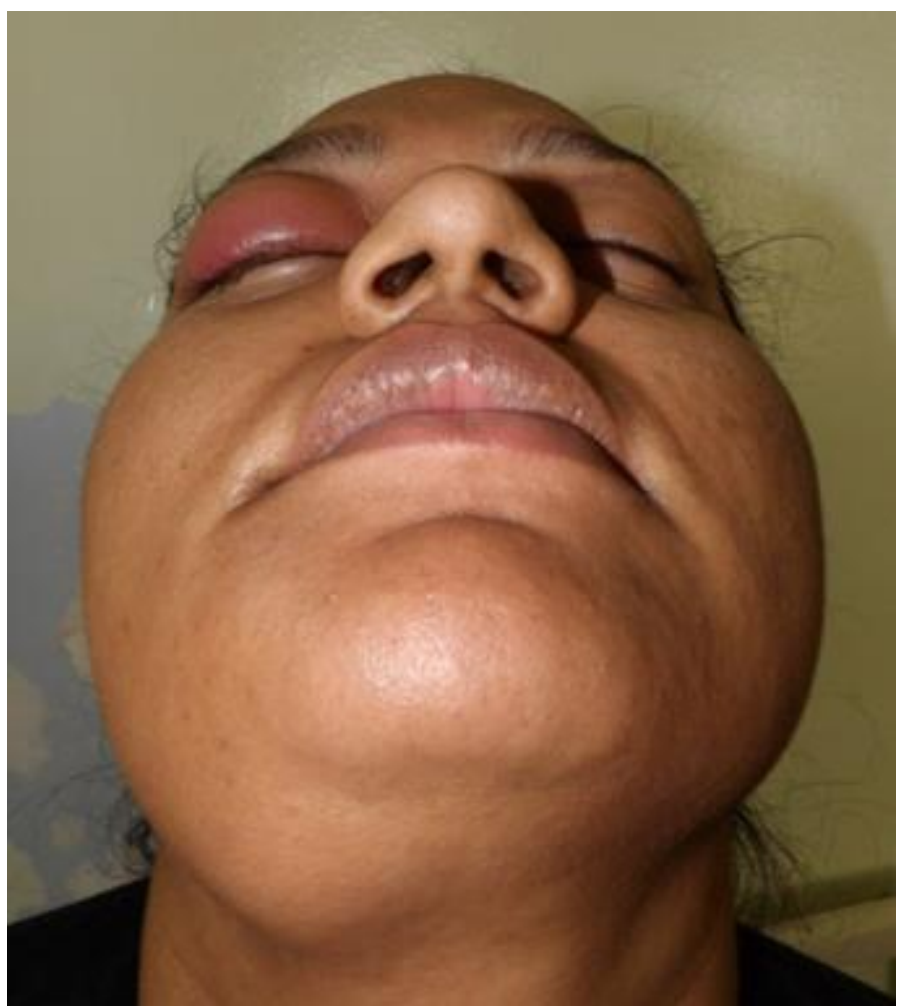

Figura 2: Aumento volumétrico evidente em região submandibular à direita.

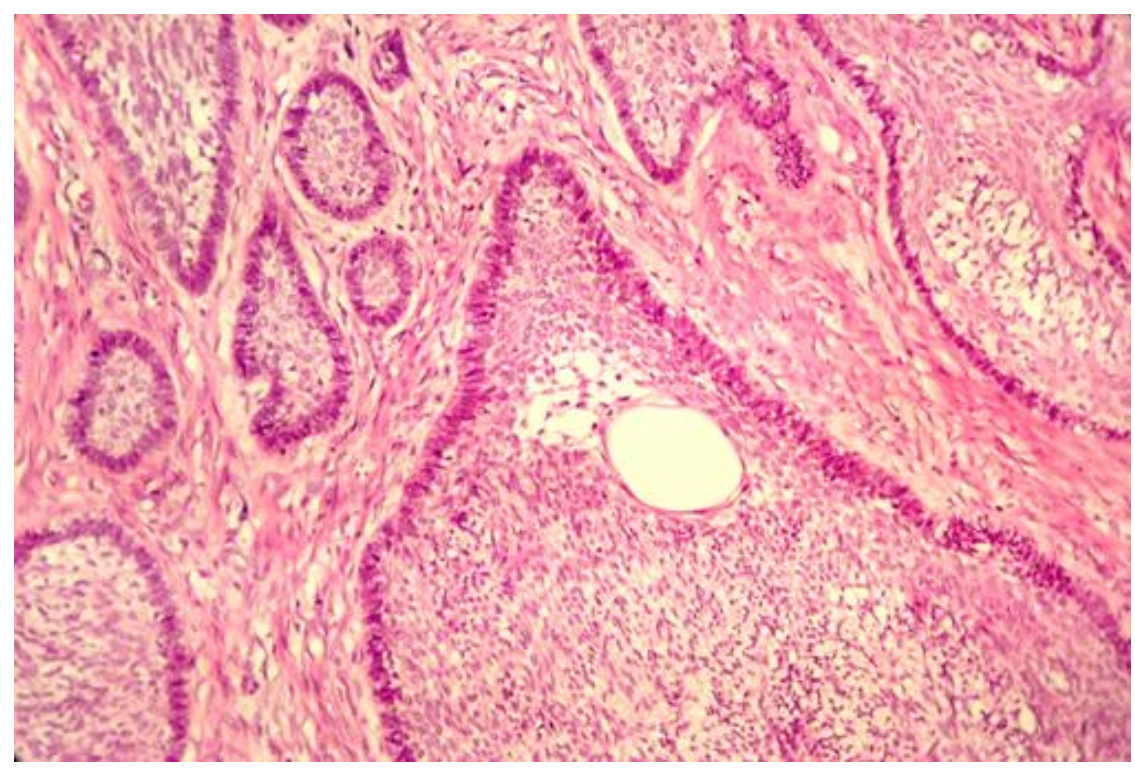

Figura 3: Fotomicrografia do ameloblastoma. Notar neoplasia composta por blocos sólidos de células basaloides com citoplasma frouxo no centro e células colunares em paliçadas.
O estudo radiográfico revelou uma lesão radiolúcida com margens escleróticas bem definidas, multilocular, com cerca de cinco centímetros na sua maior extensão, na região de corpo e ramo mandibular direito, com reabsorção radicular dos dentes 44,45 e 46 . Na tomografia computadorizada foi possível analisar melhor a extensão da lesão e a perfuração de corticais vestibular e lingual (Figura 4).
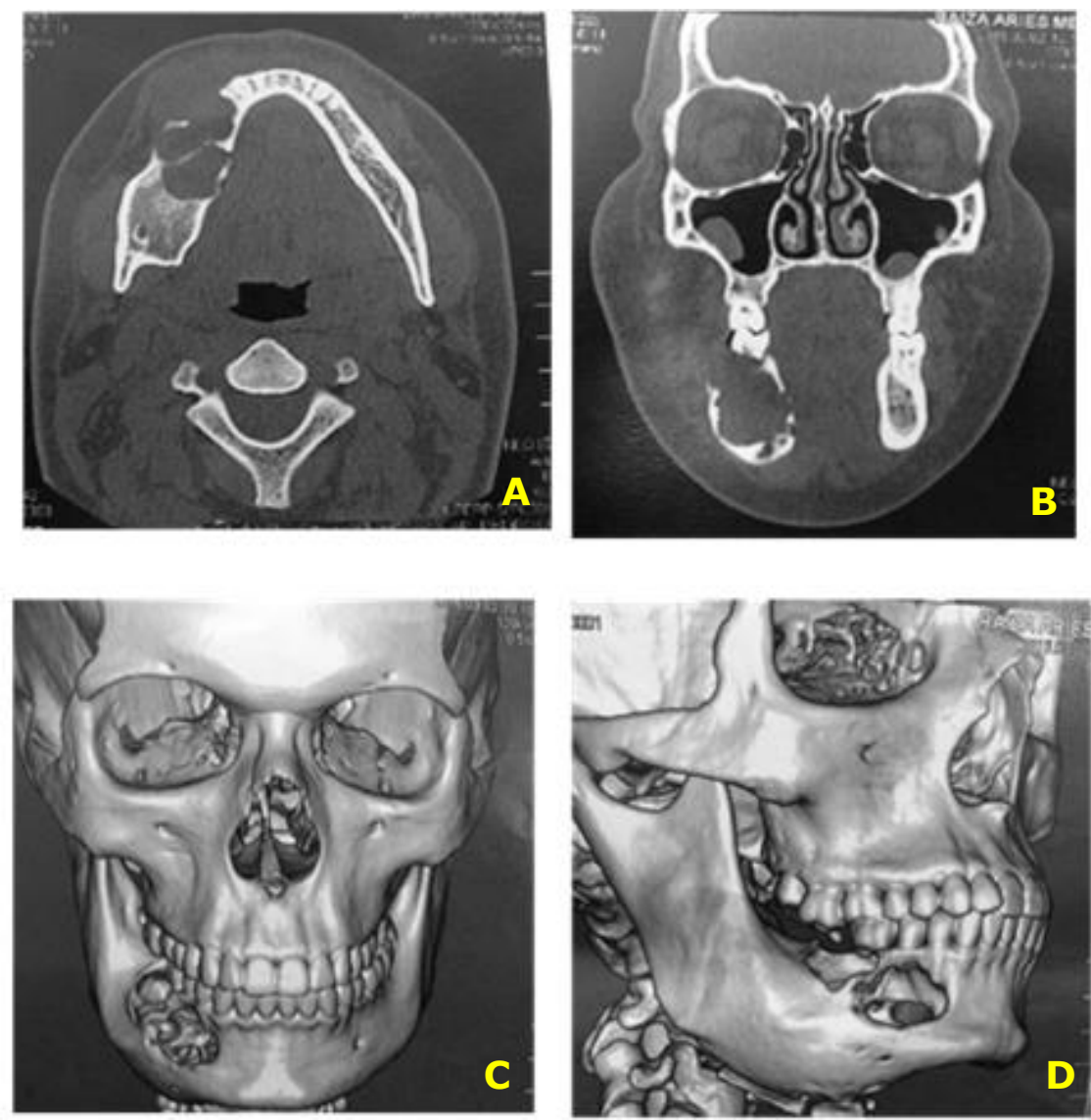

Figura 4: A e B - Tomografia computadorizada em corte axial e corona evidenciando a expansão das corticais ósseas.

C e D - Reconstrução 3D da tomografia computadorizada destacando a erosão óssea em corpo e ramo mandibular (setas).

Diante do diagnóstico definitivo e considerando-se que a paciente já havia sido submetida a inúmeros procedimentos cirúrgicos, planejou-se uma ressecção segmentar com margem de segurança de $1 \mathrm{~cm}$ e reconstrução imediata com enxerto ósseo de crista ilíaca não vascularizado e placa de reconstrução do sistema de fixação 2.4 moldada previamente à cirurgia em mandíbula seca e parafusos de titânio. Sob anestesia geral, foi instalado um bloqueio maxilomandibular para manutenção da oclusão e por meio do acesso submandibular estendido expôs-se a área de sínfise a ramo mandibular ipsilateral acometida pela lesão e iniciou-se a ostectomia em sínfise com broca 702 e serras reciprocantes (Figura 5).

Após a remoção da lesão, o enxerto foi ajustado e posicionado, sendo imobilizado por ação compressiva da

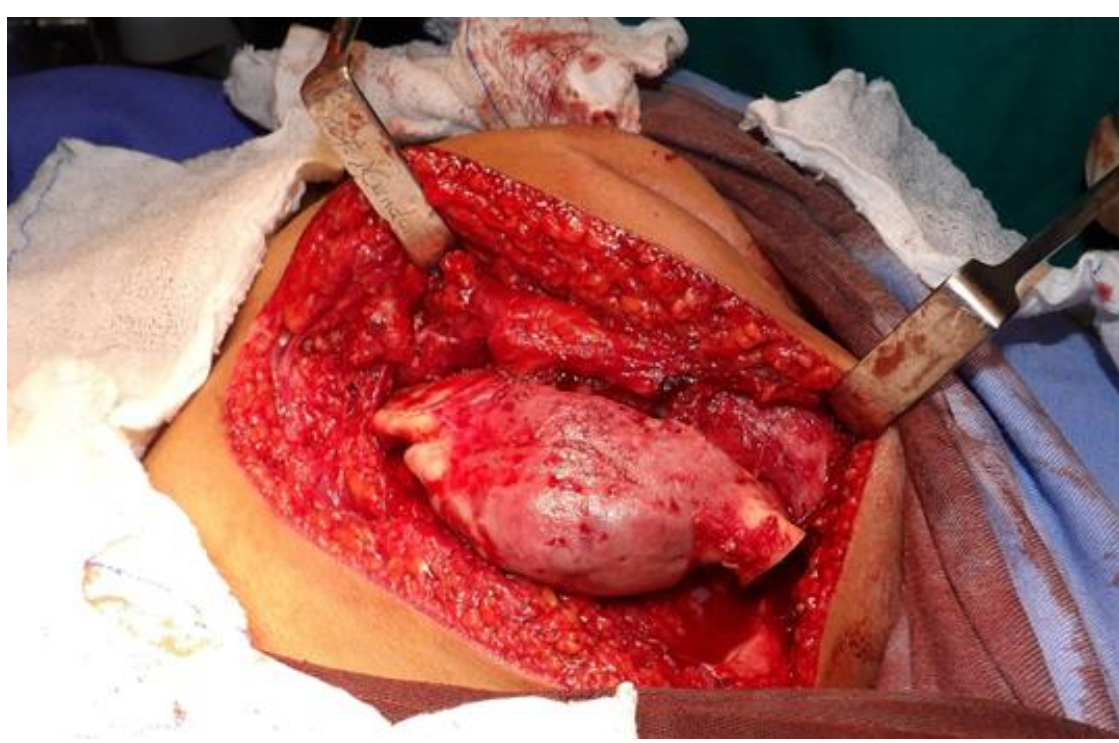

Figura 5: Acesso submandibular estendido e exposição do tumor. Início da ostectomia em sínfise. 
placa de reconstrução com parafusos bicorticais (Figura 6). Suturas por planos com Vicryl ${ }^{\mathrm{R}} 4.0$ foram realizadas para fechamento do acesso submandibular estendido e finalizado com nylon 5.0. A paciente recebeu alta hospitalar no terceiro dia pós-operatório, sem qualquer intercorrência.

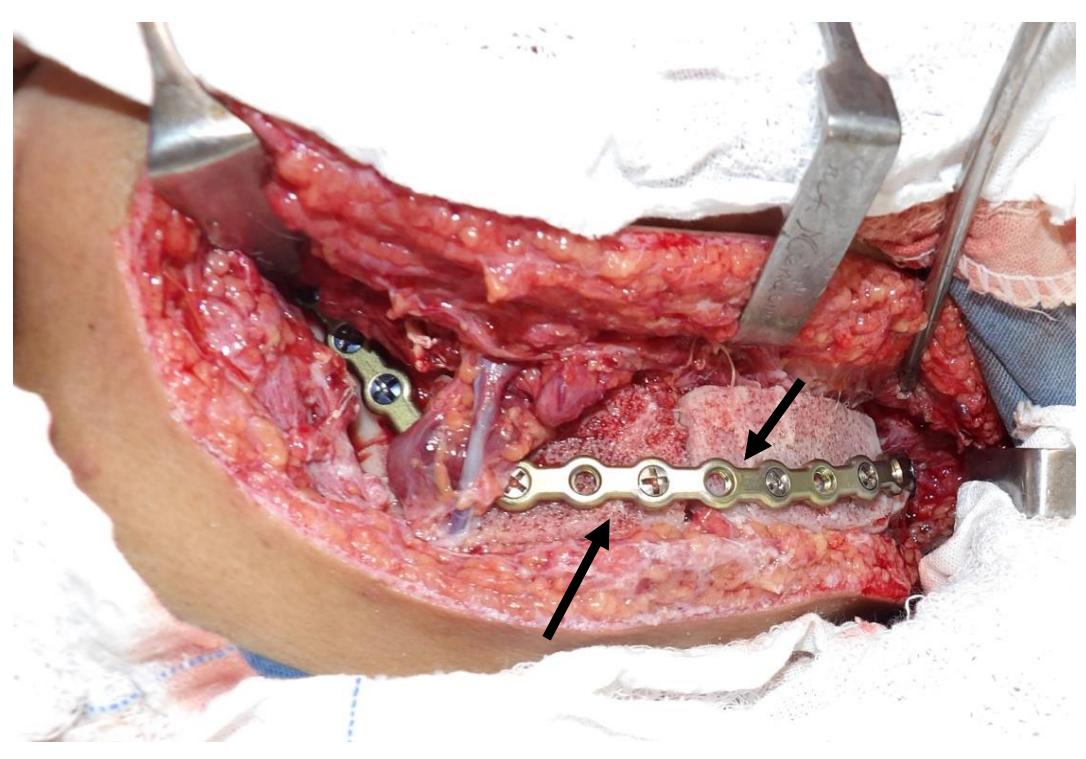

Figura 6: Aposição do enxerto de crista ilíaca fixado na placa de reconstrução do sistema $2.4 \mathrm{~mm}$ e preservação da artéria e veia facial. Notar a boa adaptação do enxerto ósseo autógeno (setas).

Através da análise de tomografia computadorizada do pós-operatório imediato pode-se observar um satisfatório contorno e simetria da face obtida com a placa de reconstrução e a estável adaptação do enxerto de crista ilíaca (Figura 7).

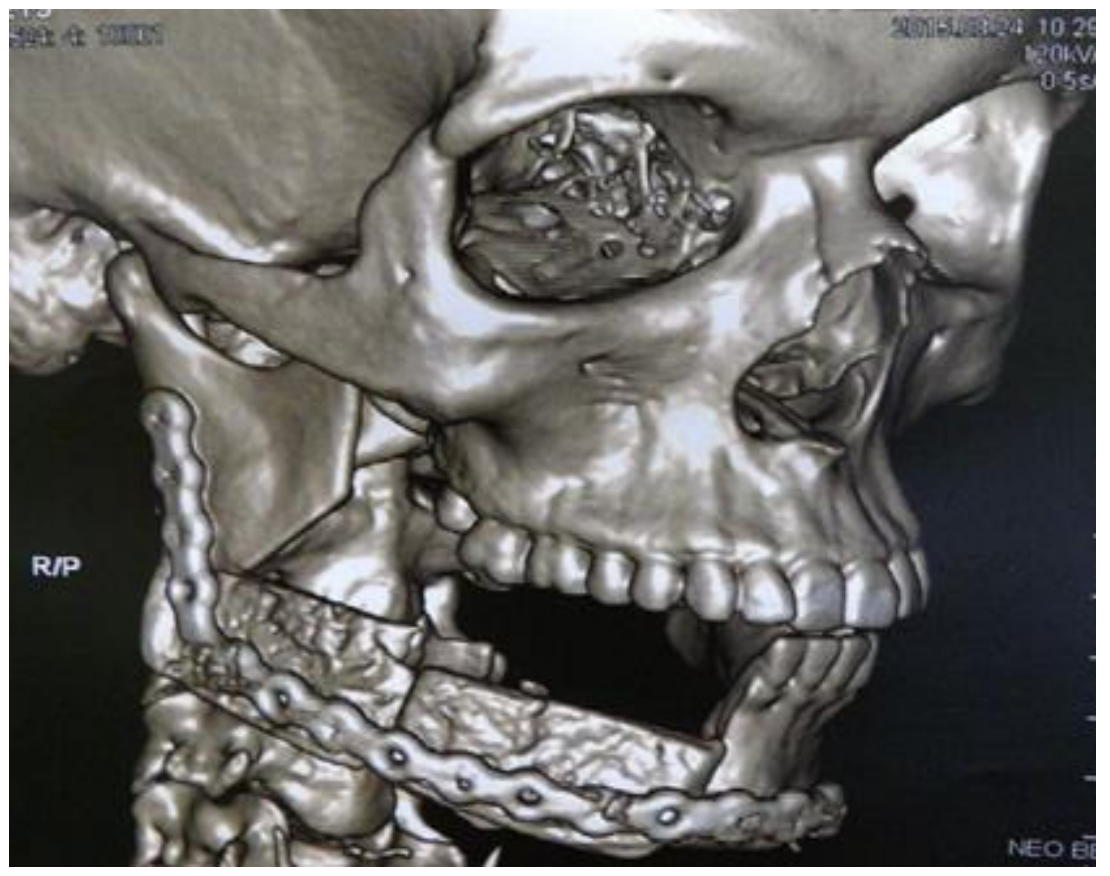

Figura 7: Reconstrução 3D evidenciando boa adaptação do enxerto na placa de reconstrução.

No retorno de pós-operatório de 120 dias constatou-se simetria facial, com bom contorno mandibular, bons aspectos de cicatrização da incisão extra-oral, mucosas íntegras, oclusão satisfatória e exame tomográfico com discreta reabsorção do volume e contorno do enxerto ósseo autógeno (Figura 8).

\section{DISCUSSÃO}

A escolha do tratamento se dá em função do tamanho, tipo de lesão, localização anatômica e evolução clínica do tumor $^{18}$. Para a conduta radical, a ressecção segmentar deve incluir uma margem cirúrgica óssea de cerca de 1 a $2 \mathrm{~cm}$ até a desarticulação ${ }^{19}$. O caso apresentado trata-se de um ameloblastoma sólido ou multicístico com $5 \mathrm{~cm}$ em região posterior de mandíbula, com rápida evolução clínica e sintomático, para o qual se indicou a ressecção segmentar, por se tratar de uma lesão que já havia sido tratada por métodos conservadores sem bons resultados, associado à reconstrução imediata com enxerto de crista ilíaca não vascularizado.

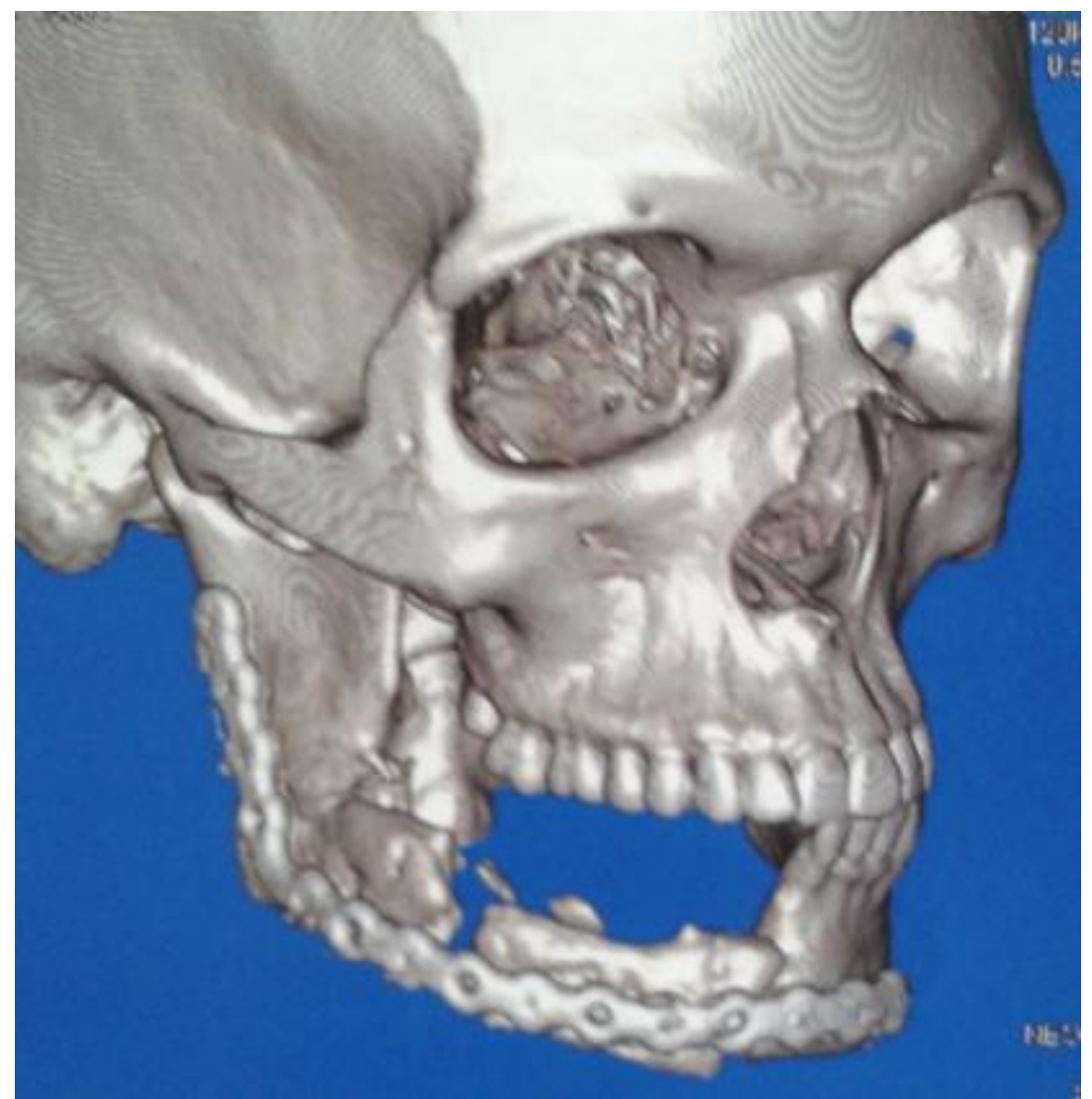

Figura 8: Reconstrução do P.O de 120 dias com

discreta reabsorção óssea, principalmente entre os enxertos.

O ameloblastoma possui um importante potencial de recidiva, se não for completamente removido ${ }^{20}$ tal fato é evidente principalmente em lesões multiloculares que apresentam índices de recidiva consideravelmente maiores que os uniloculares ou unicísticos. Deste modo, a melhor forma de tratamento ainda é controversa já que tal patologia tende a se infiltrar entre as trabéculas ósseas do osso esponjoso na periferia da lesão, antes de uma reabsorção óssea e daí tornarem-se evidente radiograficamente.

A literatura atual ainda cita fatores como tamanho, tipo de lesão, localização e histopatologia para nortearem a escolha da melhor terapêutica ${ }^{21}$. Deste modo, como conduta cirúrgica, a mandibulectomia empregada deve, de acordo com a literatura, incluir uma margem cirúrgica óssea de 1 a 2 $\mathrm{cm}$ associada à reconstrução com enxertos ósseos a fim de evitar deformidades estéticas e funcionais, seja mastigatória ou mesmo de deglutição e fonação, fatores que nortearam a terapêutica aqui empregada com sucesso até então ${ }^{22}$.

$\mathrm{O}$ melhor método de reconstrução a ser empregado varia de acordo com o tamanho do defeito. Defeitos mandibulares com mais que $5 \mathrm{~cm}$ de extensão de acordo com Foster et al. ${ }^{17}$ tendem a trazer um maior índice de complicações pós-operatórias, deixando para os defeitos maiores a necessidade de retalhos microcirúrgicos de fíbula ou crista ilíaca. Fato esse corroborado pelo presente caso clínico, uma vez que peça enviada ao histopatológico apresentou $5 \mathrm{~cm}$ em sua maior extensão.

\section{CONCLUSÃO}

O tratamento cirúrgico do tumor odontogênico queratocístico com transformação ameloblástica por meio de reconstrução imediata com enxerto autólogo de crista ilíaca é uma opção para a reconstrução de defeitos cirúrgicos mandibulares. Acreditamos que a reconstrução imediata após uma ressecção segmentar com margem de segurança é a melhor alternativa de tratamento dos ameloblastomas 
multicísticos, já que tenta promover ao máximo a remoção do tumor, reabilitação estética e funcional do paciente no mesmo procedimento cirúrgico.

\section{REFERÊNCIAS}

1. Philipsen HP. In: Barnes L, Eveson JW, Reichart P, Sidransky D, eds. World Health Organization classification of tumours. Pathology and genetics of head and neck tumours. Lyon (France): IARC; 2005. p. 3067.

2. Neville BW, Damm DD, Allen CM, Bouquot JE. Patologia Oral \& Maxilofacial. $2^{\mathrm{a}}$ ed. Rio de Janeiro: Guanabara Koogan; 2004. 705p.

3. Tamme T, Tiigimäe J, Leibur E. Mandibular ameloblastoma: a 28-years retrospective study of the surgical treatment results. Minerva Stomatol. 2010;59(11-12):637-43.

4. Hertog D, Bloemena E, Aartman Ih, van-der-Waal I. Histopathology of ameloblastoma of the jaws; some critical observations based on a 40 years single institution experience. Med Oral Patol Oral Cir Bucal. 2012 Jan 1;17 (1):e76-82.

5. Bataineh AB. Effect of preservation of the inferior and posterior borders on recurrence of ameloblastomas of the mandible. Oral Surg Oral Med Oral Pathol Oral Radiol Endod 2000; 90(2):155-63.

6. Chapelle Kaom, Stoelinga PJW, De wilde PCM, Brouns JJA, Voorsmit RACA. Rational approach to diagnosis and treatment of ameloblastomas and odontogenic keratocysts. Br J Oral Maxillofac Surg. 2004; 42(5):38190.

7. Baker A, Mcmahon J, Parmar S. Immediate reconstruction of continuity defects of the mandible after tumor surgery. J Oral Maxillofac Surg 2001;59(11):1333-9.

8. Disa JJ, Cordeiro PG. Mandible reconstruction with microvascular surgery. Semin Surg Oncol 2000;19(3):226-34.

9. Dhanuthai K, Chantarangsu S, Rojanawatsirivej S, Phattarataratip E, Darling M, Jackson-Boeters L, et al. Ameloblastoma: a multicentric study. Oral Surg Oral Med Oral Pathol Oral Radiol. 2012;113(6):782-8.

10. Piazza, J.L. Contribuição ao estudo do tratamento do ameloblastoma. RGO Rev Gaúcha Odontol. 1984; 32(1):27-30.

11. Arita ES, Pereira MF, Jaeger RG, Nosé AS, Freitas A. Transformação ameloblástica de um cisto odontogênico. Rev Odontol Univ São Paulo. 1990; 4(1):76-80.

12. Lima GM, Nogueira RLM, Rabenhorst SHB. Detecção da proteína p53 em cistos odontogênicos e ameloblastoma unicístico. Rev Cir Traumatol BucoMaxilo-Fac. 2006; 6(1): 47-54.

13. Mendonça JCG, Masocatto DC, Oliveira MO, GaettiJardim EC, Coelho TMK, Terra GAP, et al. Enxerto ósseo de mento estabilizado em pré-maxila e reabilitação com implantes osseointegrados:relato de caso. Arch Health Invest. 2015; 4(1):13-19.

14. Matsumoto MA, Filho HN, Francischone AE, Consolaro A. Microscopic analysis of reconstructed maxillary alveolar ridges using autogenous boné grafts from the chin and iliac crest. Int J Oral Maxillofac Implants 2002;17(4):507-16.

15. Ahlmann E, Patzakis M, Roidis N, Shepherd L, Holtom P. Comparison of anterior and posterior iliac crest boné harvest in terms of harvest site morbidity and functional outcomes. J Bone Joint Surg Am. 2002;84-A(5):716-20.
16. Pogrel, M. A. et al. A comparison of vascularized and nonvascularized bone grafts for reconstruction of mandibular continuity defects. J Oral Maxillofac Surg. 1997; 55(11):1200-6.

17. Foster RD, Anthony JP, Sharma A, Pogrel MA. Vascularized bone flap versus nonvascularized bone grafts for mandibular reconstruction: an outcome analysis of primary bony union and endosseous implant success. Head Neck. 1999; 21(1):66-71.

18. Vasan NT. Recurrent Ameloblastoma in an Autogenous Bone Graft After 28 Years: a Case Report. N Z Dent J 1995; 91(403):12-3.

19. Feinberg SE, Steinberg B. Surgical Management of Ameloblastoma. Current Status of the Literature. Oral Surg Oral Med Oral Pathol Oral Radiol Endod 1996;81(4):383-8.

20. Gomes ACA, Dias E, Gomes DO, Paraíso DP, Nascimento GJF, Cabral RAA. Ameloblastoma: Tratamento cirúrgico conservador ou radical? Rev Cir Traumatol Buco-Maxilo-Fac. 2002; 2(2):17-24.

21. Tawfik MA, Zyada MM. Odontogenic tumors in Dakahlia, Egypt: analysis of 82 cases. Oral Surg Oral Med Oral Pathol Oral Radiol Endod. 2010; 109(2):e6773.

22. Carlson ER, Marx RE. Mandibular Reconstruction Using Cancellous Cellular Bone Grafts. J Oral Maxillofac Surg 1996; 54(7):889-97.

\section{CONFLITO DE INTERESSES}

Os autores declaram não haver conflitos de interesse.

\section{AUTOR PARA CORRESPONDÊNCIA}

Ellen Cristina Gaetti Jardim

ellengaetti@gmail.com
Submetido em 29/07/2016 Aceito em 05/12/2016 